\title{
A COMPACT RESIDUAL GAS IONIZATION PROFILE MONITOR (RGIPM) SYSTEM ${ }^{*}$
}

\author{
W. C. Sellyey and J. D. Gilpatrick, Los Alamos National Laboratory, Los Alamos, NM
}

\begin{abstract}
The Accelerator Production of Tritium (APT) and Spallation Neutron Source (SNS) accelerators will produce high power density beams, which cannot be observed using conventional intercepting beam profile devices. The beam produces electrons when it ionizes the residual gas in the beam tube. These electrons, when accelerated by a uniform electric field and guided by uniform parallel magnetic field, produce a projected image of the beam on a detector perpendicular to the fields. A particle tracking program shows that this can be done with a resolution better than the needed $100 \mu \mathrm{m}$. There are typically no dipoles available in these accelerators to produce the needed magnetic field. Triple dipole systems are being designed which will give no net trajectory change to the beam, but will produce the needed field for the diagnostic. Although the field is not completely uniform in the electron collection region, particle tracking calculations show that the resolution is not seriously degraded relative to a uniform field. Several ways of viewing the beam profile are considered. Radiation resistant and hardened materials needed for this are discussed .
\end{abstract}

\section{INTRODUCTION}

RGIPM has been implemented by detecting either the positive ions or the electrons that result from the beam ionizing the residual gas in the beam tube. Here we will consider only electrons. This paper will discuss design considerations for the space restricted, high radiation environment of a high power linac. It will also discuss various ways of imaging the profile information contained in the electrons coming from the ionized residual gas. These RGIPM are intended to observe 200 to $1700 \mathrm{MeV}$ proton or $\mathrm{H}$ - beams, with $\mathrm{cw}$ current up to $100 \mathrm{~mA}$. The beam profiles will be non-Gaussian with dimensions down to $0.8 \mathrm{~mm}$ rms.

\section{RESOLUTION LIMIT OF RGIPM METHOD}

One method of implementing RGIPM is to use a strong magnetic field so that the resolution is determined by the Larmor radius of the electron. This will be one of the ways investigated here. A second method is to take advantage of the fact that the Larmor period of nonrelativistic electrons depends only on the magnetic field

\footnotetext{
"Work supported by the U. S. Department of Energy.
}

strength. In this case all electrons can be made to go through nearly one complete orbit in the magnetic field and the resolution can be much smaller than the Larmor radius [1]. Thus lower magnetic fields can be used. A Monte Carlo particle tracking code was used to investigate the achievable resolution [2]. It takes into account the beam space charge and the distribution of the initial electron velocities. A minor modification of the program enabled the calculation of the delta function response (particle distribution at the detector for electrons coming from a plane parallel to a plane defined by the beam direction and the magnetic field.) From this the resolution can be calculated.

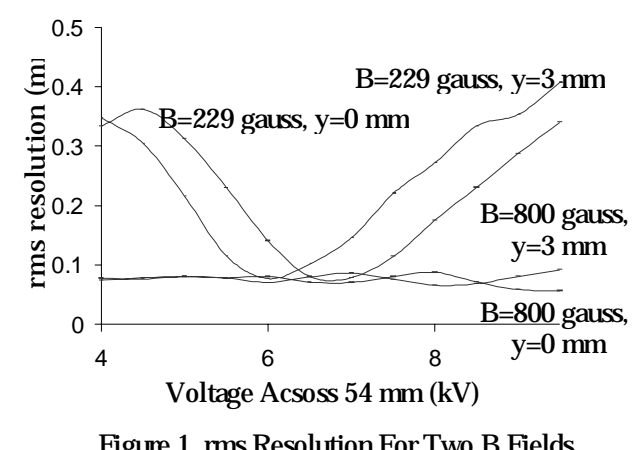

Figure 1 shows the calculated rms delta function response for a gap of $54 \mathrm{~cm}$ and a gap voltage range of 4 to $9.5 \mathrm{kV}$. Two curves are for a B-field of 229 gauss, and two are for 800 gauss. Within each pair, one is for the beam centerd on the beam tube, and the second is with the beam displaced $3 \mathrm{~mm}$ towards the detector (beam width $=1 \mathrm{~mm} \mathrm{rms}$ ). This illustrates the main features of the two ways of generating the beam profile at the electron detector. With the 800 gauss field, the resolution is independent of beam position and electric field and is expected to vary in proportionally to the magnetic field. In the 229 gauss case, the needed field is much lower but the resolution depends strongly on the fields and beam position. In practice, the magnetic fields will not be uniform because short dipoles will be used.

\section{MAGNET DESIGN}

Triple dipoles are being designed using a commercial finite element code (Vector Fields, TOSCA). Both coil and permanent magnet driven designs are being investigated. Figure 2 shows one quadrant of a permanent magnet design for a $16 \mathrm{~cm}$ beam tube. The 
beam moves in the $\mathrm{z}$ direction and the ionization electrons drift in the $y$ direction. The center of the magnet, marked by + , is $4 \mathrm{~cm}$ above the beam tube center (origin). The magnet extends $+/-24 \mathrm{~cm}$ in the $\mathrm{x}$ direction. Figure 3 shows $\mathrm{B}_{\mathrm{y}}$ along the $\mathrm{y}$ axis, and this is near where most of the observed electrons will be moving. It also shows $B_{y}$ along a line parallel to the $z-$ axis through the center of the magnet.

\section{RADIATION FIELDS}

A major challenge will be to use imaging devices which can survive the expected high radiation levels. Assuming that the average beam loss is $0.1 \mathrm{nA} / \mathrm{m}$, the expected dose rate at $1 \mathrm{~m}$ from the beam line at $1.1 \mathrm{GeV}$ will be $20 \mathrm{rad} / \mathrm{h}$ [3]. Beam line component activation calculations indicate that for the safety of personnel working on the beam line, dose rates will need to stay well below this level. Beam loss rates in LANSCE between $300 \mathrm{MeV}$ and $800 \mathrm{MeV}$ were measured to average about $0.2 \mathrm{nA} / \mathrm{m}$ [4].
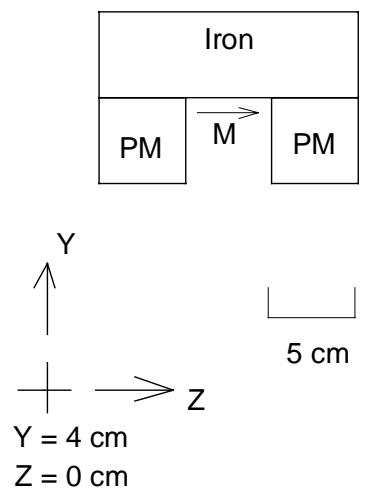

Figure 2. One quadrant of a triple dipole using permanent magnet (PM). The arrow points in the magnetisation $(\mathrm{M})$ direction in the iron.

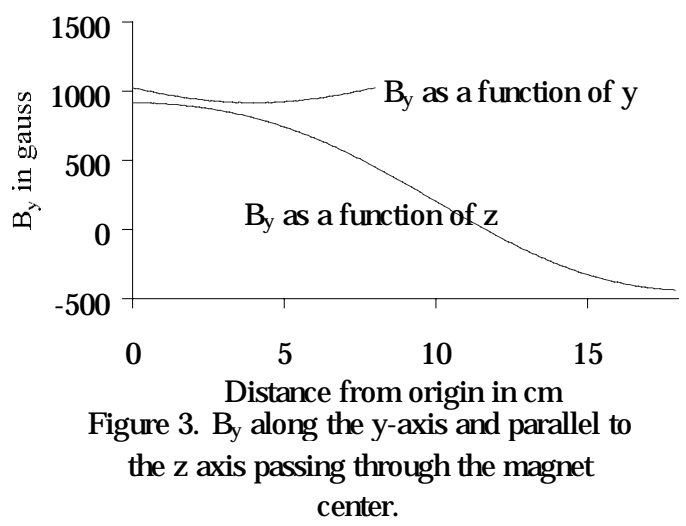

These losses were deemed excessive and would be too high for SNS and APT. Thus a maximum average of 20 $\mathrm{rad} / \mathrm{h}$ at $1 \mathrm{~m}$ will be assumed in this paper. This translates into $0.175 \mathrm{Mrad} /$ year at $1 \mathrm{~m}$ and $7 \mathrm{Mrad} /$ year at $2.5 \mathrm{~cm}$ from the beam.

\section{RADIATION RESISTANT AND HARDENED ITEMS}

Some of the radiation resistant materials and components under consideration for use are listed below:

Measurements have been made on the light attenuation caused by radiation damage to sapphire[5]. An initial darkening will happen in 75 hours under nominal operating conditions (assuming $10 \mathrm{krad} / \mathrm{h} \gamma$ 's). No further darkening due to $\gamma$ 's will occur. It will take about $4 \bullet 10^{4}$ years for neutrons to cause a $50 \%$ light attenuation in a 1 $\mathrm{cm}$ thick sapphire $2.5 \mathrm{~cm}$ from the beam.

High $\mathrm{OH}$ content silica fibers $1 \mathrm{~m}$ long will take 70 years to reduce transmission by $37 \%$ in the visible spectrum [6].

Cerium activated Yttrium Aluminum garnet (YAG:Ce) is a linear scintillator which has been measured to half its light output in $10000 \mathrm{~h}$ with a $10 \mathrm{keV}$ electron beam of current density $71 \mathrm{nA} / \mathrm{cm}^{2}$ [7]. Light output is about 18 photons $/ \mathrm{keV}$ [8]. Resolution and electron depth penetration is 20 microns at $100 \mathrm{keV}$ [9]. Thus at $5 \mathrm{keV}$ these would be about 5 microns. It has been found that $0.2 \mathrm{mC}$ of $15 \mathrm{MeV} 5 \mathrm{~mm}$ diameter electron beam on 1 $\mathrm{mm}$ thick YAG produced no visible darkening [10]. From this one can estimate that it would take $>40$ Mrad to cause a $50 \%$ reduction in light transmission of a 0.1 $\mathrm{mm}$ thick piece of YAG.

Micro Channel Plates (MCP) have been exposed to 1 Mrad of Co-60 $\gamma$ 's with no degradation in performance [11]. Thus one can estimate that MCP's will be useable with total dose $>10$ Mrad. Gains of several 1000 can be obtained with one MCP and over $10^{8}$ with stacks of MCP's. Output currents for conventional $25 \mathrm{~mm} \mathrm{MCP}$ is around $2 \mu \mathrm{A}$. Advanced devices can output $20 \mu \mathrm{A}$ and have a life of $40 \mathrm{C} / \mathrm{cm}^{2}$.

Channeltrons are electron multiplying tubes about 100 $\mu . \mathrm{m}$ across. They are made of the same material and in a similar fashion as MCP's and thus the radiation related life times are expected to be similar. Gains are up to $10^{8}$ and output currents up to $5 \mu \mathrm{A}$.

Resistive position sensing element are made by depositing a roughly rectangular, uniform thickness, resistive surface on alumina. The effect of accumulated dose on this element is expected to be unimportant.

A radiation hardened CID camera manufactured by CIDTEC is specified as operating to $1 \mathrm{Mrad}$ without change in performance. It is a standard RS-170 camera. Noise is about 1400 electrons per pixel per frame.

Xybion has combined an intensifier with the CIDTEC camera. The intensifier resolution is better than $88 \mu \mathrm{m}$ FWHM. One photo electron produces $2 \cdot 10^{5}$ photons at the intensifier output. There is a reduction of 1.46 by the fiber coupling and the quantum efficiency of the CID is about $20 \%$. For the integrated count over all light 
receiving pixels $\mathrm{S} / \mathrm{N} \approx 5$. This may allow the observation of a single photo electron generated at the photo cathode and the determination of its impact point. For high radiation use, the photo cathode and phosphor substrates and fiber coupling may need to be high $\mathrm{OH}$ silica.

Radiation hardened charge sensitive pre amplifiers that can withstand $>100 \mathrm{krad}$ are available. For these to survive several years, the radiation will be attenuated to $5 \mathrm{krad}$ per year by placing them $2 \mathrm{~m}$ away, $1 \mathrm{~m}$ into the accelerator concrete shielding [12]. A possible choice for this amplifier is the Amptek A225 which is rated at 100 krad. Noise (rms) will be 1600 electrons with $3 \mathrm{~m}$ of cable on the input. Typical easily achievable count rates are $10 \%$ s.

\section{IMAGING SYSTEMS}

Some candidates for imaging the electron current from the beam and associated considerations are:

The electrons hit a $0.1 \mathrm{~mm}$ thick YAG crystal, and form an image viewed by an intensified camera about 1 $\mathrm{m}$ away. At a pressure of $10^{-6}$ torr, a $1 \mathrm{GeV}, 1 \mathrm{~mm} \mathrm{rms}$, 100 ma proton beam, will cause a YAG crystal to loos $10 \%$ of its light output in 6 years. The view port would probably be sapphire. At $10^{-8}$ torr, $1 \mathrm{~mA}$ beam will produce $5 \cdot 10^{7}$ photons $/(\mathrm{s} \bullet \mathrm{cm})$. Assuming $50 \%$ transmission, a 2 in lens $1 \mathrm{~m}$ from the YAG would produce 4000 photons $/(\mathrm{s} \bullet \mathrm{cm})$ on the intensifier. Assuming 20\% quantum efficiency this would result in 800 events per second on the CID. Thus a good profile could be obtained in 1 to 10 seconds. Alternately, if the 2 in lens has a magnification of 0.5 and the beam rms width is $1 \mathrm{~mm}$, the summed $\mathrm{S} / \mathrm{N}$ for $1 \mathrm{~cm}$ of beam when imaged on the CID for one second will be about 80. The error in a Gaussian width would be $5 \%$. Achromatic lenses made from sapphire and fused quarts or Cassegrain optics would be used to generate an image on the intensifier. Light collection efficiency 5 times larger than assumed here is probably achievable. The life of the system should be 5 years or greater.

A stack of three $18 \mathrm{~mm}$ MCP's would be places about $2.5 \mathrm{~cm}$ from the beam. The MCP output for each electron strike would land on a resistive sheet. Four outputs at the corners of the sheet would be amplified by radiation shielded charge sensitive amplifiers. Each output would produce about $2.5 \cdot 10^{6}$ electrons. The amplifier noise of 1600 electrons will be unimportant compared to the error caused by fluctuations in gain of MCP channels. The signals would be digitised and positions calculated with $70 \mu \mathrm{m}$ rms accuracy. This system would be purchased from a vendor. At a pressure of $10^{-10}$ torr $\left(\mathrm{N}_{2}\right)$ the peak electron flux for a $1 \mathrm{~mm} \mathrm{rms}$ $100 \mathrm{~mA}$ proton beam is estimated to be $4 \bullet 10^{6} /\left(\mathrm{s}^{\bullet} \mathrm{cm}^{2}\right)$. To reduce the count rate to $10000 / \mathrm{s}$ a mask with a 100 $\mu \mathrm{m}$ slot will be placed in front of the MCP. The peak electron flux that a conventional MCP can handle in this type of position measuring device has been measured as
$10^{7} /\left(\mathrm{s}^{\bullet} \mathrm{cm}^{2}\right)$ [13]. An advanced MCP may be able to handle ten times this rate. Thus an important consideration for this system will be the pressure in the beam line and a vacuum pump will probably need to be part of the system. Radiation damage may limit the life of this detector to a couple of years.

A Channeltron would be mechanically moved across the e-beam. It would have a mask cut with a $100 \mu \mathrm{m}$ by $300 \mu \mathrm{m}$ slot in front of it. A charge sensitive amplifier would be remotely placed and shielded and electrons would be individually counted. The Channeltron can handle $\mathrm{MHz}$ count rates and thus the count rate will be limited by the charge sensitive amplifier. At $10^{-10}$ torr the count rate would be about $1.2 \mathrm{kHz}$. Radiation damage may limit the life of this system to a couple of years. The mechanical drive and radiation hard position sensing system for this unit will need to be a couple of feet from the Channeltron, outside the magnetic field.

A $100 \mu \mathrm{m}$ optical fiber (silica or sapphire) would conduct light from a $100 \mu \mathrm{m}$ diameter, $100 \mu \mathrm{m}$ long YAG scintillator to a high gain photo multiplier tube a couple of meters from the beam tube. Typically 5 photons would reach the photo multiplier for every electron on the scintillator.

\section{REFERENCES}

[1] Private communication, A. Jason, Los LANL, Los Alamos, N.M.

[2] A. Hahn, FNAL, Batavia, IL

[3] Private communication, Eric Pitcher, LANL, Los Alamos, N.M.

[4] N. Bultman, A. Jason, E. Pitcher, G. Russel, W. Sommer, D. Weinacht, R. Woods, "Los Alamos Next-Generation Spallation Source Volume I", LANL Report LA-UR-95-4300, (1995) p. 2-81.

[5] P. W. Levy, "Color Centers and Radiation-Induced Defects in $\mathrm{Al}_{2} \mathrm{O}_{3}$ ", Phys. Rev. 123, (1961) 1226.

[6] D. W. Cooke, B. L. Bennett, E. H. Farnum, "Optical Absorption of Neutron-Irradiated Silica Fibers", Journal of Nuclear Materials 232 (1996) 214.

[7] R. Autrata, P. Schauer, Jos. Kvapil, J. Kvapil, "Singel-Crystal Aluminates - A New Generation of Scintillators for Scanning Electron Microscopes and Transparent Screens in Electron Optical Devices", Scanning Electron Microscopy, 11 (1983) 489.

[8] W. S. Graves, E. D. Johnson, S. Ulc, "A High Resolution Electron Beam Profile Monitor and its Applications", AIP Conference Proceedings, 451 (1998) 206.

[9] M. Kotera, Y. Kamiya, "Computer Simulation of Light by HighEnegy Electrons in YAG Single Crystals", Ultramicroscopy, 54 (1994) 293.

[10] J. G. Power, N. Barov, M. E. Conde, W. Gai, R. Konecny, P. Schoessow, "Initial Characterisation of the YAG Crystal" AGN \#32, Technical Report, Argonne National Laboratory (1997).

[11] J. G. Timothy, R. L. Bybee, "Effects of 1-MeV Gamma Radiation on a Multi-Anode Microchannel Array Detector Tube", Rev. Sci. Instrum., 50 (1979) 743.

[12] K. Tesch, "A Simple Estimation of the Lateral Shielding for Proton Accelerators in the Energy Range 50 to $100 \mathrm{MeV}$ ", Radiation Protection Dosimetry, 11 (1985) 165.

[13] Private communication, M. R. Mellon, Quantar Technology Inc., Santa Cruz, CA. 